\title{
Mid-infrared nanometallic antenna assisted silicon waveguide based bolometers
}

\author{
Yangbo Wu, ${ }^{\dagger}$ Zhibo Qu, ${ }^{\dagger}$ Ahmed Osman, ${ }^{\dagger}$ Wei Cao, ${ }^{\dagger}$ Ali Z. Khokhar, ${ }^{\dagger}$ Jordi \\ Soler Penades, ${ }^{\dagger}$ Otto L. Muskens, ${ }^{\ddagger}$ Goran Z. Mashanovich, ${ }^{\dagger}$ and Milos \\ Nedeljkovic ${ }^{*, \dagger}$ \\ $\dagger$ Optoelectronics Research Centre, University of Southampton, Southampton, Hampshire, \\ SO17 1BJ, UK \\ $\ddagger$ School of Physics and Astronomy, University of Southampton, Southampton, Hampshire, \\ SO17 1BJ, UK \\ ISchool of Electrical Engineering, University of Belgrade, 11120 Belgrade, Serbia
}

E-mail: M.Nedeljkovic@soton.ac.uk

\section{Abstract}

The mid-infrared (MIR) wavelength region is attracting more and more research for applications such as medical diagnostics, environmental monitoring, and free space communications. In the MIR, thermal detectors play an important role because they can operate over a large wavelength range, can be fabricated using CMOS compatible processes, and do not require cooling. Today no other MIR detector technology is able to fill this gap. We demonstrate the first uncooled silicon waveguidebased bolometers, in the Silicon-on-Insulator (SOI) and suspended silicon waveguide platforms. The bolometers comprise gold plasmonic antennas on the waveguide surface that heat up when they absorb light, and amorphous silicon thermometers (formed by ion implantation), whose electrical resistance changes by $0.90 \pm$ $0.26 \% \mathrm{~K}^{-1}$ when they are heated. We show that suspending the bolometers improves their performance, and achieve sensitivities of up to $1.13 \pm 0.04 \%$ change in resistance per milliwatt of input power, with a noise equivalent power of $66 \mu \mathrm{W} / \sqrt{\mathrm{Hz}}$. Calculations suggest the NEP could in future, be further reduced by 4 orders of magnitude.

\section{Keywords}

Mid-infrared, Silicon Photonics, Sensor, Detector, Bolometer, Antenna, Amorphous Silicon

\section{Introduction}

The MIR spectrum $(2-16 \mu \mathrm{m})$ has great potential to be used in many different fields, such as medical diagnostics, environmental monitoring, industrial process control and free space communications. $\frac{112}{}$ In these applications, sensing attracts much attention, because many molecules exhibit intense and unique absorption features in the MIR wavelength region. Silicon photonics, also known as group IV photonics, has become widely used for near-infrared (NIR) integrated photonics in the last two decades, particularly for data communications applications. Silicon and germanium are transparent at up to $8 \mu \mathrm{m}$ and $16 \mu \mathrm{m}$ respectively, therefore there is an opportunity to extend the wavelength range of silicon photonics into the MIR to create integrated lab-on-a-chip sensing systems.

Development of passive group-IV devices for MIR has advanced rapidly such as SOI rib and strip waveguides, $\stackrel{3}{3}$ and suspended $\mathrm{Si}$ waveg- 
uides. ${ }^{4}$ To extend to wavelengths beyond the transparency range of $\mathrm{Si}$, waveguides based on Ge on $\mathrm{Si}, \stackrel{5}{\mathrm{SiGe}^{6}}$ and suspended Ge platforms ${ }^{7}$ have been investigated. Meanwhile there has also been progress in developing on-chip light sources; a quantum cascade laser operating at $4.8 \mu \mathrm{m}$ has been integrated with silicon waveguides, $\frac{8}{8}$ and supercontinuum generation in SiGe waveguides at up to $8 \mu \mathrm{m}$ has been reported. ${ }^{9}$ Detectors also play an important role in MIR photonic systems. So far, Si defect, 10 III-V, 11 graphene, ${ }^{12 \mid 13}$ and black phosphorus ${ }^{14}$ MIR photodetectors have all been integrated with silicon waveguides. In addition, GeSn has also been applied in MIR detection. $\frac{15}{15}$ However, aside from graphene the operating wavelength ranges of these photodetectors are limited by the bandgaps of the absorbing materials. Furthermore, due to the thermal excitation of electrons, photodetectors usually require cooling down to reduce the noise. In the case of graphene and black phosphorus, they are not currently manufacturable at wafer scale.

Thermal detectors are an alternative solution for infrared detection. Bolometers are a type of photodetector in which light is absorbed and converted to heat, and the resulting temperature change alters the bolometer's electrical resistance, which can be measured by monitoring a bias current. Bolometers can work at room temperature, and the absorption mechanism can be tailored to operate at a range of wavelengths. In fact, today the market for commercial uncooled MIR imaging cameras is dominated by microbolometers, which absorb light over a very broad wavelength range, do not need to be cooled, and are fabricated using CMOS processes, often at the back-end-of-line on top of a CMOS read out circuit. While the ultimate sensitivity and frequency bandwidth of bolometers are generally both worse than those of conventional photodetectors, integrating microbolometers with silicon photonic waveguides will be an excellent solution for many MIR integrated sensors, where uncooled operation and affordable fabrication will be more important than the detector speed, provided that the sensitivity is sufficient.

In a bolometer in which periodic incident ra- diation is absorbed to change the temperature of the material, the temperature change can be expressed as: $\stackrel{16}{ }$

$$
\Delta T=\frac{\varepsilon \Phi_{0}}{\left(G_{t h}^{2}+\omega^{2} C_{t h}^{2}\right)^{1 / 2}}
$$

where $\Delta T$ is the temperature difference, $\varepsilon$ is the absorbing efficiency of detector, $\Phi_{0}$ and $\omega$ are the optical power amplitude and the modulating frequency of incident radiation, $C_{t h}$ is the thermal capacitance of the detector and $G_{t h}$ is the thermal conductance to the surroundings. The resistance of the material is then influenced by the temperature change, which can be described as a function of the ambient temperature by the temperature coefficient of resistance (TCR).

$$
T C R=\frac{d R}{R} \frac{1}{d T}
$$

where $R$ is the resistance, $d R$ is resistance change, and $d T$ is the temperature change. A simple way to improve the performance of bolometers is selecting a material with a high TCR. Vanadium oxide $\left(V O_{x}\right)$ is the most common thermometer material in bolometers. $V O_{x}$ deposited by reactive ion beam sputtering and then annealed has achieved a high TCR of 6.5 $\% / \mathrm{K}{ }^{17}$ Amorphous silicon (a-Si:H) is another common thermometer material with a TCR of $2.5 \% / \mathrm{K}, \frac{18119}{1}$ which also has the advantage of being fully CMOS compatible. Amorphous germanium-silicon ( $\mathrm{a}-\mathrm{Ge}_{x} \mathrm{Si}_{y}: \mathrm{H}$ ) has also been developed as a thermal sensing film in bolometers. Compared to a-Si:H, it has a higher TCR of $4.3 \% / \mathrm{K}$ and lower resistance. ${ }^{20}$ Aside from these traditional 3D materials, carbon nanotubes (with a TCR of $0.3 \% / \mathrm{K}$ ) and the $2 \mathrm{D}$ material graphene (with a TCR of 4-11 \%/K) have been applied in bolometers, 2122 although the fabrication is more challenging.

Enhancing the absorbing efficiency is another way to improve the bolometer performance. A common method used in out of plane coupled thermal camera microbolometers is to use a reflector below the bolometer to form a resonant optical cavity ${ }^{23}$ that enhances the electric field at the absorber. In addition, optical antennas that can activate surface plasmon polaritons 
(SPPs) to confine the incident radiation have also been used to improve the absorption efficiency. $\frac{21 / 24}{2 n}$ this paper, we demonstrate the first MIR silicon waveguide-based bolometers with optical antennas as absorbers and amorphous silicon as the thermometer.

\section{Design \& Simulation}

We designed two types of waveguide-based bolometers, both of which are shown in Figure 1 . The designed bolometers are based on silicon waveguides with a sub-wavelength grating lateral cladding of the kind demonstrated in 25 (and with the same dimensions), so that the waveguide can be suspended by etching away the silicon dioxide lower cladding, and thus the thermal conductance to the surroundings can be reduced. Since silicon is transparent from $1 \mu \mathrm{m}$ to $8 \mu \mathrm{m}$, we design $\mathrm{Au}$ optical antennas acting as plasmonic absorbers on the waveguide surface. The optical antennas consist of three $\mathrm{Au}$ nanorod antennas. Light incident from the waveguide will excite a half-wavelength antenna resonance mediated by SPPs on the gold nanorod. The electric field at the ends of antennas is enhanced and the light is confined in this region. This light is absorbed by the antennas and temperature will be increased. After that, the heat is conducted to the waveguide and to the thermometer arms. In our designs the plasmonic antennas were offset by $1 \mu \mathrm{m}$ from the thermometer arms because these arms will result in more scattering instead of absorption in the antennas from the simulations. The thermometer is made from amorphous silicon, which is created from crystalline silicon by high dose germanium ion implantation. $\frac{26127}{}$ The amorphous silicon arms are thinned to make sure that the whole depth of the Si can be amorphized in one ion implantation step to simplify the fabrication. Then, the resistance change of amorphous silicon is measured via the contact pads on either side of the amorphous silicon arms. To avoid any leakage current through the crystalline silicon, there are trenches surrounding the contact pads. In the first design (B1), the waveguide crosses the amorphous sili- con arms and leads to an output surface grating coupler. In the second device variation (B2), the waveguide is terminated after the end of the bolometer in order to reduce the thermal conductance and capacitance, and hence increase the temperature change. To aid alignment of light into the waveguide, the B2 design includes a multi-mode interferometer (MMI) acting as a 1x2 splitter before the bolometer, from which the second output waveguide leads to a grating coupler that can be used to help optimise the input light coupling.

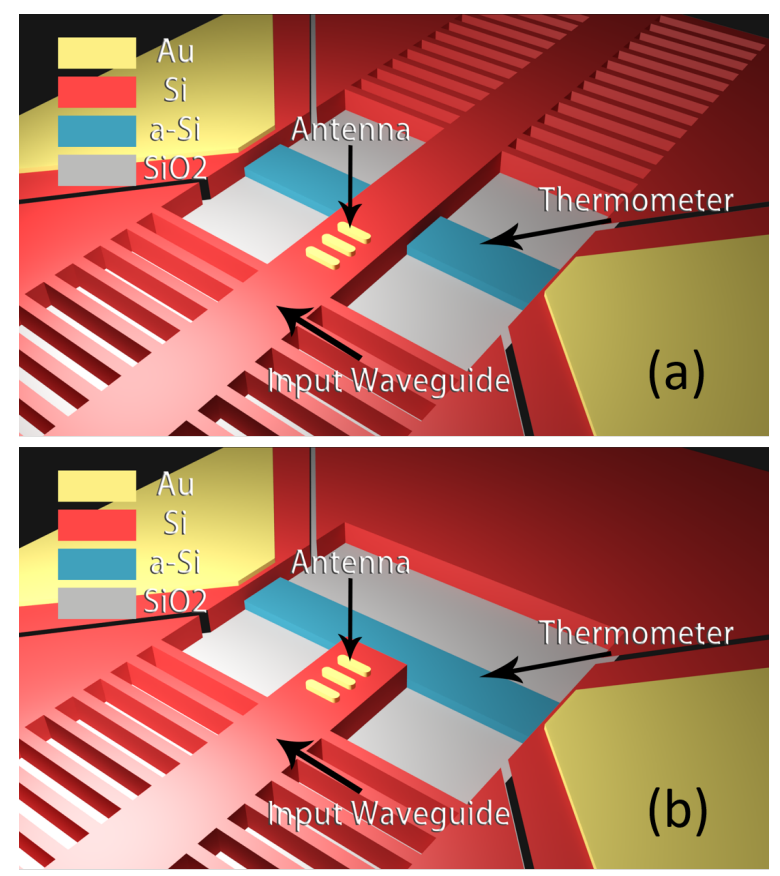

Figure 1: Structure diagrams of the designed bolometers based on (a) straight waveguides (B1) and (b) cut straight waveguides (B2).

To estimate the performance of the antennas on waveguide, we used a 3D FDTD solver to simulate the response. In this simulation the antennas are positioned on a suspended silicon waveguide. The width and thickness of the waveguide are $1.3 \mu \mathrm{m}$ and $500 \mathrm{~nm}$, respectively. The designed waveguide supports only the fundamental TE mode. The near-field electric field profile of three rod antennas are shown in Figure 2(a). The designed antennas comprise three separated rod antennas. Each antenna has a $470 \mathrm{~nm}$ length, $1600 \mathrm{~nm}$ width, $20 \mathrm{~nm}$ thickness, and there is a $400 \mathrm{~nm}$ gap between them. In rod antennas, the travelling-wave SPPs are 
reflected at the antenna ends which sets up a half-wavelength resonance with a charge distribution that peaks at the end caps of the antenna, resulting in strong confinement of light at these regions. ${ }^{28}$ Since the metal exhibits a large absorption coefficient, the confined light will be absorbed strongly. When the antenna number increases from one to three and these antennas are close to each other, the scattering, reflection and transmission from one antenna can interact with the other two antennas. So, these antennas are not independent and the interaction between them greatly enhances the absorption, as was shown in several other works. 2931

To compare different materials, antennas made from various materials have been simulated. Figure 2(c) compares the fraction of energy absorbed, scattered, reflected, and transmitted at $3.8 \mu \mathrm{m}$ wavelength by a group of three antennas, for a selection of plasmonic materials. Of these common materials, Cr, Pt, Ti and TiN do not achieve high absorption. Among the rest of the materials, $\mathrm{Al}$ and $\mathrm{Ta}$ oxidise in air; $\mathrm{Al}$ and Ag can be etched by HF (and are therefore incompatible with the fabrication process); $\mathrm{Cu}$ can easily diffuse into silicon and change its optical and electrical properties. However, gold is stable when it is exposed to air, and it can survive in HF. Gold is therefore the best candidate for the antennas in this device. In addition, compared with a single gold antenna, three gold antennas shows much stronger absorption while transmission, scattering, and reflection are suppressed.

\section{Fabrication}

Figure 3 shows the fabrication process. Firstly, the thermometer arms are implanted with Ge ions to damage the crystalline silicon and create amorphous silicon. The implant energy and dose are $190 \mathrm{keV}$ and $1 \times 10^{15}$ ions $/ \mathrm{cm}^{2}$. Before the ion implantation, the crystalline silicon is patterned and etched down to $200 \mathrm{~nm}$ by an Inductively Coupled Plasma (ICP) tool. This is done because in order to create a $500 \mathrm{~nm}$ thick a-Si layer, Ge ions would require a high implant
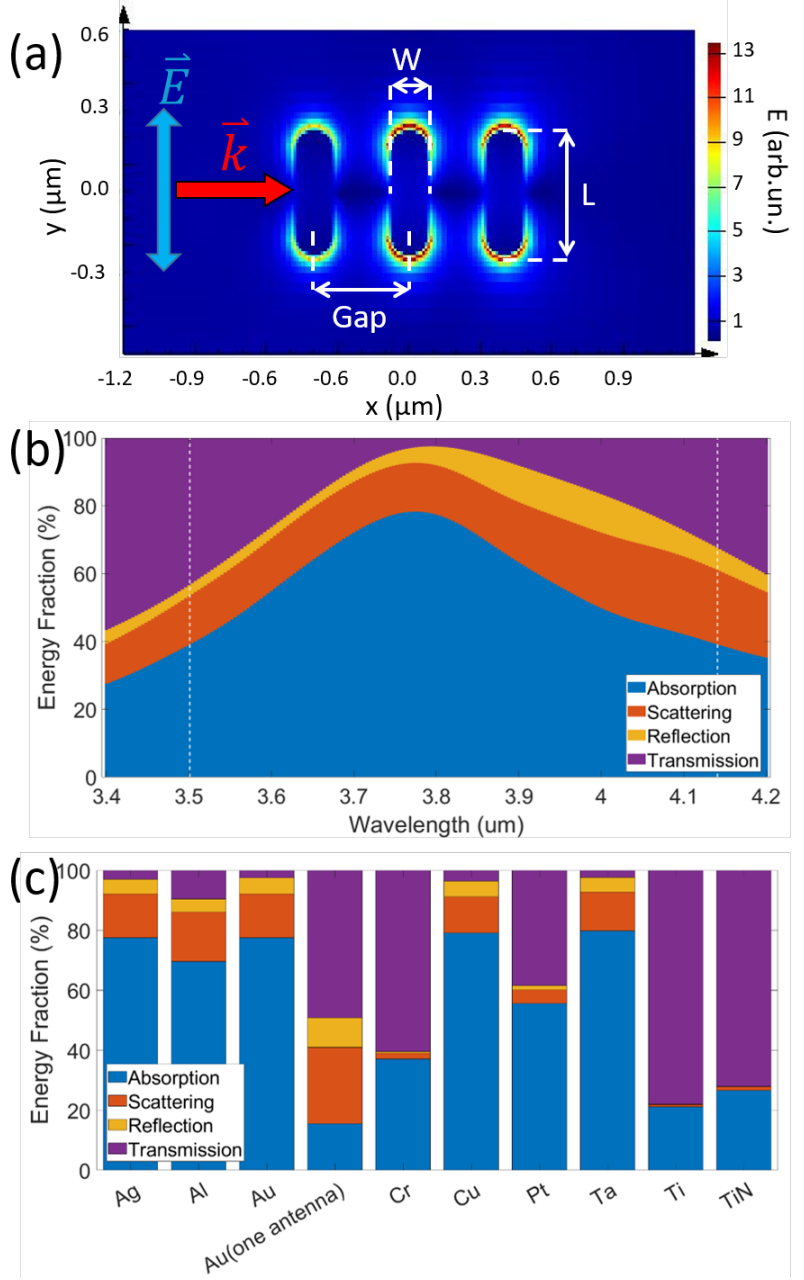

Figure 2: (a)Near-field electric field profile of 3 rod antennas simulated by $3 \mathrm{D}$ FDTD.(b)Energy fraction spectrum of the $3 \mathrm{Au}$ antennas with $470 \mathrm{~nm}$ length, $160 \mathrm{~nm}$ width, 20 $\mathrm{nm}$ thickness and $400 \mathrm{~nm}$ gap. The absorption 3 - $\mathrm{dB}$ bandwidth is $640 \mathrm{~nm}$. (c)Energy fraction of optimized three antennas with different materials at $3.8 \mu \mathrm{m}$ wavelength. 
energy which would make them go through the resist and damage the un-patterned crystalline silicon. To use a thicker resist would influence the precision of lithography. After that, the waveguides, gratings, and trenches surrounding contact pads are etched by ICP. The next step is patterning and depositing $1 \mathrm{~nm}$ of chromium as an adhesion layer and $20 \mathrm{~nm}$ of gold for optical antennas using an e-beam evaporator. The chip is then left in 1-methyl-2-pyrrolidone (NMP) for lift-off. Finally, 100 nm thick Au contact pads with a $10 \mathrm{~nm}$ Cr adhesion layer are deposited and lifted off by the same method.
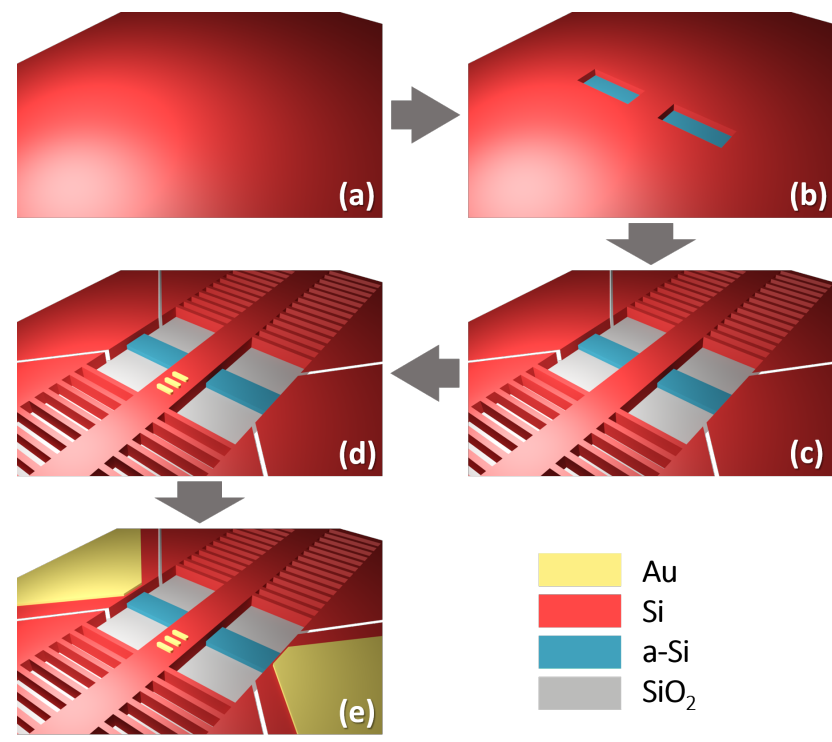

Figure 3: Fabrication process of the waveguidebased bolometers. (a) Begin with a SOI chip with $500 \mathrm{~nm}$ thick Si layer. (b) Etch the thermometer arms to $200 \mathrm{~nm}$ and implant them with Ge ions to create a-Si. (c) Dry etch subwavelength cladding waveguides and gratings. (d) Deposit optical antennas on the waveguides by e-beam evaporation. (e) Deposit Au contact pads by e-beam evaporation.

To further improve the performance of the bolometers, some of the B1 type devices were suspended by etching the silicon dioxide layer with $45 \%$ HF vapor - these suspended devices are referred to as "B1S" devices in the following sections. During HF etching the antennas are protected with a patterned layer of photoresist. After 90 minutes etching, the $3 \mu \mathrm{m}$ silica box underneath the waveguide has been fully etched. Figure 4 shows an SEM image of the bolometer with suspended waveguide.

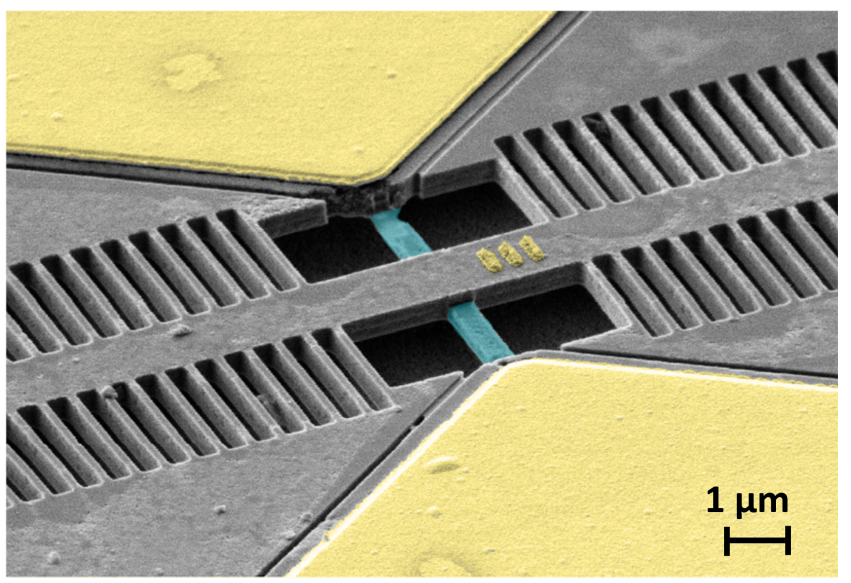

Figure 4: SEM image of the bolometer based on the suspended waveguide. The $3 \mu \mathrm{m}$ silica BOX has been fully etched. Yellow regions are gold and blue regions are a-Si.

\section{Experimental Results}

\section{Optical Antennas}

The optical response of the antennas was first characterised to verify that they work as absorbers when patterned on top of a waveguide. In practice the optical antennas not only absorb the incident light, but also scatter and reflect it. However, due to the limitations of our experimental setup, only the transmission can be measured. The transmission spectra of different antennas have been measured and normalized to the transmission of identical test waveguides without antennas. It should be noted that the actual dimensions of the antennas are different than the designed values and the three antennas in the array are often not identical. The antennas designed to have a length of $500 \mathrm{~nm}$ and width of $160 \mathrm{~nm}$ are in practice broadened to $620 \mathrm{~nm}$ length and $250 \mathrm{~nm}$ width, respectively. In addition, there is a variation in the dimension of antennas of $\pm 10 \mathrm{~nm}$, creating uncertainty in their actual dimensions. Therefore, in all places where we refer to the dimensions of the experimental antennas, we have taken the nominal antenna dimensions and corrected them by the average experimental dimension 
bias error measured from scanning electron microscope (SEM) images. Figure 5(a) shows the transmission spectrum of $620 \mathrm{~nm}$ long antennas, and Figure 5(b) plots the transmissions of antennas with different actual lengths at a central wavelength of $3.8 \mu \mathrm{m}$. The plasmonic antenna resonance has a bandwidth larger than the available spectral range of the QCL, therefore it is impossible to directly identify the plasmon resonance lineshape. Narrowband features in Figure 5(a), such as the weakly pronounced maximum at $3830 \mathrm{~nm}$, are attributed to other wavelength dependent components in the waveguide system. For the dependence on antenna length, the transmission initial shows an initial high transmission for the shortest antennas followed by a decrease in transmission to values in the range $20 \%$ to $30 \%$ over the 570 $650 \mathrm{~nm}$ antenna length range. This behaviour is consistent with resonant antenna as discussed below. The flat response over a wide range of lengths illustrates that the antenna response is quite robust with respect to variations in the antenna length. Overall, the antenna transmissions are somewhat higher than predicted by the simulations, which we attribute to the difference between the nominal and actual antenna shapes.

Since the silicon waveguide and grating only support the TE mode, we cannot measure the performance of the antennas with a TM mode. The wavelength range of the transmission measurement is also limited by the laser emission range and grating coupler transmission spectrum. To clearly show the resonance nature of the antenna absorption, we therefore fabricated antenna arrays on bulk silicon in which each unit has three antennas, so that we could investigate the antenna response over a wider wavelength range and with two different polarisations, when the light is incident from out of plane in the direction perpendicular to the array. The details are shown in Section A of the Supporting Information. The measurements confirm the resonant and polarisation dependent behaviour of the antennas, as well as the characteristic dependence of transmission on antenna length, consistent with Figure 5(b).
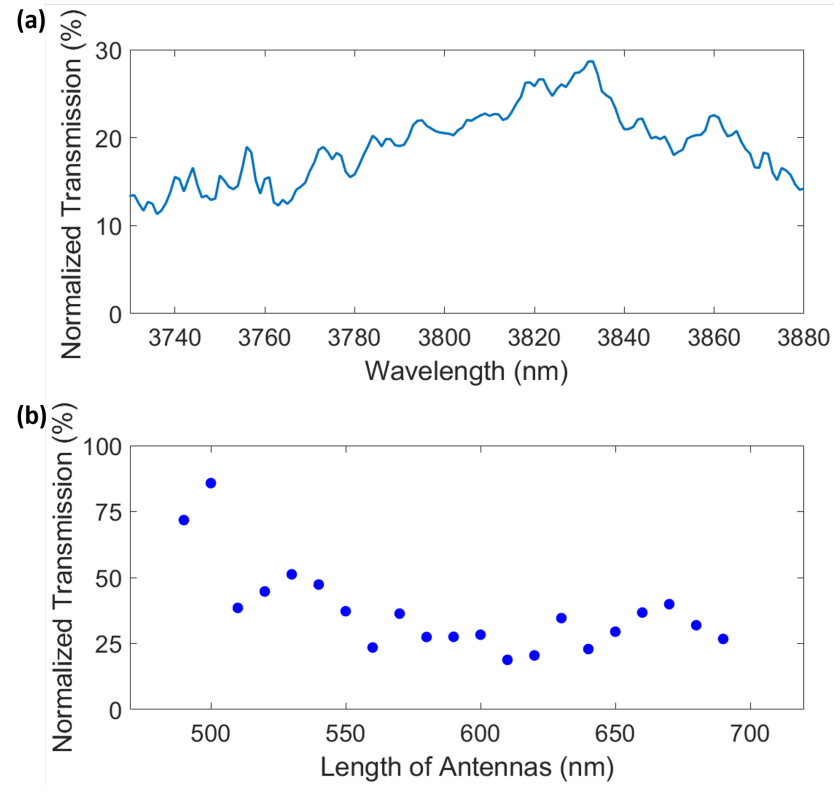

Figure 5: (a)The experimental transmission spectrum of antennas with $500 \mathrm{~nm}$ length. (b)Transmission at $3.8 \mu \mathrm{m}$ wavelength versus antenna length.

\section{Temperature Coefficient of Resis- tance of a-Si Thermometers}

Whereas the TCR of amorphous silicon films has been reported in the literature before, $18 \mid 19$ none of these values are based on the amorphous silicon created by Ge ion implantation. To measure the TCR of our devices a Peltier element beneath the sample stage is used to change the temperature of the whole chip. Once the temperature is stable, a DC source is connected across the two contact pads of a B1 bolometer to measure the IV curves of the a-Si arm. Figure 6(a) shows the IV curve of the a$\mathrm{Si}$ arm at $45^{\circ} \mathrm{C}$. The IV curve is non-linear, indicating that a Schottky contact is formed between the contact pads and silicon. We fit the slope of the IV curve to calculate the resistance. The resistance at different temperatures, normalized to the resistance at room temperature, is plotted in Figure 6(b), and the slope efficiency of the linear fitting is the TCR of the a-Si arm, which is $0.90 \pm 0.26 \% / \mathrm{K}$ from the experimental result. 


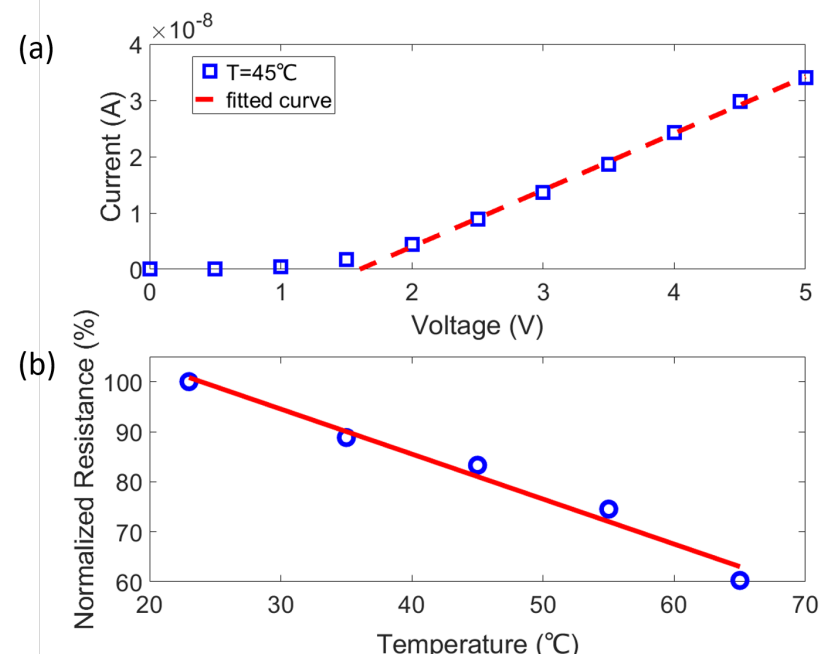

Figure 6: (a) IV curve of the a-Si bolometer arms at $45^{\circ} \mathrm{C}$ (blue line) and fit to linear section (red dashed line). (b) Fitted resistances versus temperature (blue circles) and a linear fitted curve (red line).

\section{Response of Bolometers}

In the experiment, we measured B1, B2, and B1S bolometers and show the results. A diagram of the mid-infrared measurement setup for DC device characterization is shown in Figure 7. A Daylight Solutions tunable QCL operating in the wavelength range from 3.72$3.88 \mu \mathrm{m}$ is used as the source. Light coming from the laser is modulated by a chopper wheel which is connected with a control driver (Thorlabs MC200B). The modulated light is coupled into a MIR fibre by using a ZnSe lens whose transmission range is $0.5-20 \mu \mathrm{m}$. Then the light is coupled into the chip from the fibre's cleaved end via a grating coupler. After passing through the devices under test on the chip, the light is coupled into another fibre leading to the external photodetector. After aligning the two fibres using the external photodetector, the DC source (Keithley 2401) applies a voltage to the bolometer through the two probes. A lockin amplifier (Signal Recovery 7265) is connected with the bolometer, the external photodetector, and the chopper to read out the signal. Moreover, the noise equivalent current is measured by the lock-in amplifier when the laser is turned off.

Instead of directly measuring the resistance

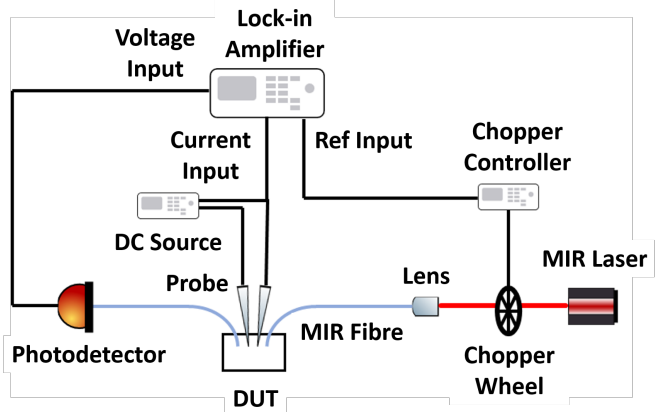

Figure 7: Mid-Infrared DC measurement setup for characterising the fabricated bolometers.

change of the bolometer, we measure the percentage change in current with a constant bias $(15 \mathrm{~V})$. The response versus the input power at $3.8 \mu \mathrm{m}$ wavelength with $400 \mathrm{~Hz}$ chopper frequency for the three bolometer types is plotted in Figure 8(a). Because some parameters in Equation 11, namely $G_{t h}, C_{t h}$, and even the TCR, change with temperature, the relationship between the bolometer response and the input power is nonlinear. However, in a small temperature range, the nonlinear effect is weak, which means we can use a linear model to simplify this relationship. From the linear fit result, the reference device, B1S without antennas show a sensitivity of $0.16 \pm 0.01$. The sensitivity of B1 at $400 \mathrm{~Hz}$ chopper frequency is $0.17 \pm 0.01 \% / \mathrm{mW}$. For B2 the sensitivity is enhanced to $0.80 \pm 0.03 \% / \mathrm{mW}$. This improvement comes from the greatly reduced thermal capacitance of the cut waveguide, $C_{t h}$ in Equation 1. For the suspended bolometer B1S, the sensitivity reaches $1.13 \pm 0.04 \% / \mathrm{mW}$, because the silicon dioxide is etched by HF vapour, and the thermal conductivity of air is far lower than the thermal conductivity of silica. Therefore, as the thermal conductance between the bolometer and its surroundings $G_{t h}$ is reduced, it results in a higher sensitivity. Table 1 presents the sensitivity of each bolometers and their references. The enhancement from antennas is up to $8.49 \mathrm{~dB}$.

The frequency response of the bolometers was also measured. The Figures 8(b) and (c) show the sensitivity and noise-equivalent power (NEP) versus the chopper frequency. In the B1 bolometer, due to the straight waveguide, 

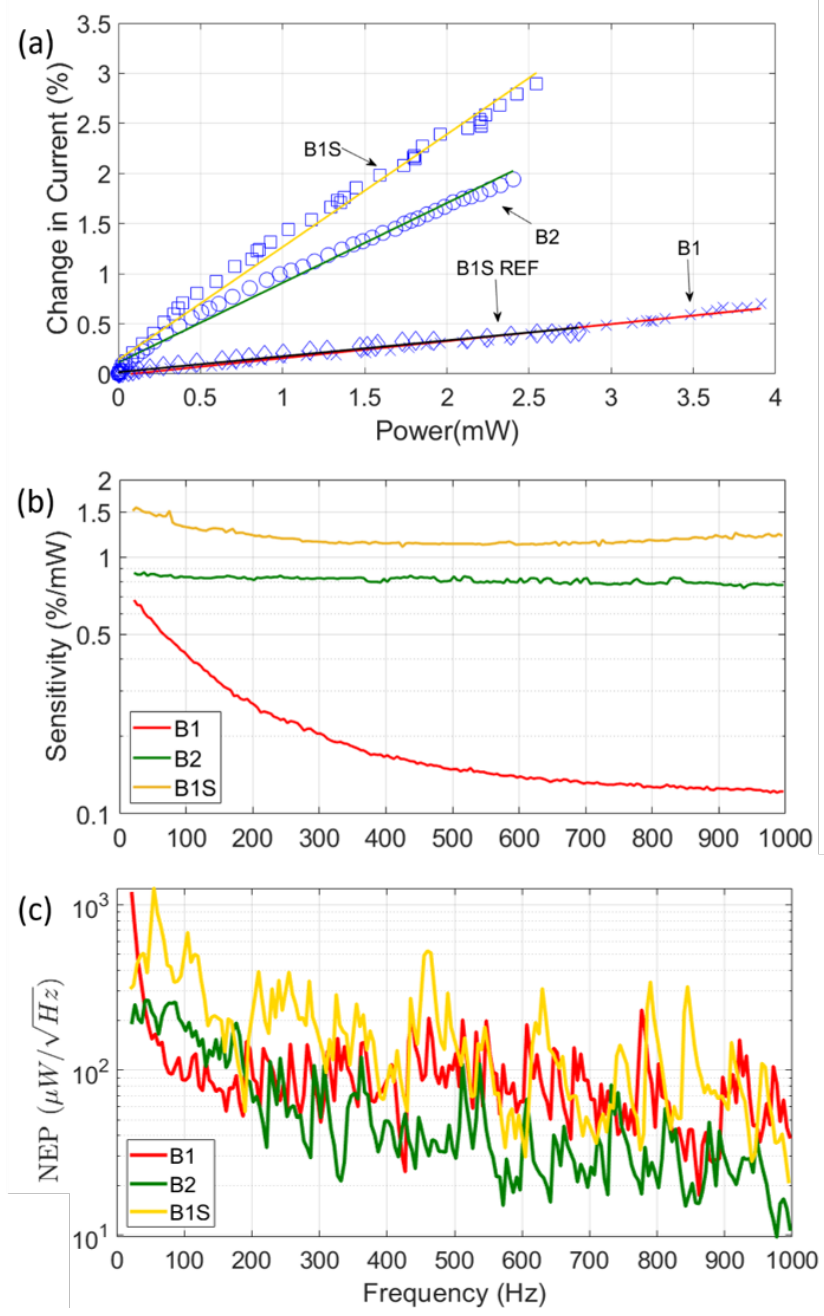

Figure 8: (a)Bolometer response versus input power at $3.8 \mu \mathrm{m}$ with $400 \mathrm{~Hz}$ chopper frequency for bolometers B1, B2, B1S and B1S REF.(b) Sensitivity and (c) NEP versus chopper frequency for three types bolometers, B1, B2 and B1S.

Table 1: Performance Parameters of Bolometer

\begin{tabular}{cccc}
\hline & \multicolumn{2}{c}{$\begin{array}{c}\text { Sensitivity } \\
(\% / \mathrm{mW})\end{array}$} & $\begin{array}{c}\text { Sensitivity } \\
\text { Enhancement }\end{array}$ \\
& Bolometer & Reference & $(\mathrm{dB})$ \\
\hline B1 & $0.17 \pm 0.01$ & $0.11 \pm 0.01$ & 1.89 \\
$\mathrm{~B} 2$ & $0.80 \pm 0.03$ & $0.21 \pm 0.01$ & 5.81 \\
$\mathrm{~B} 1 \mathrm{~S}$ & $1.13 \pm 0.04$ & $0.16 \pm 0.01$ & 8.49 \\
\hline
\end{tabular}

the large thermal capacitance requires a longer time to heat up. Therefore, the response decays faster with the chopper frequency. The $3 \mathrm{~dB}$ bandwidth of B1 is $142 \mathrm{~Hz}$, and the sensitivity of $\mathrm{B} 1$ at $20 \mathrm{~Hz}$ chopper frequency is 0.68 $\% / \mathrm{mW}$. In the $\mathrm{B} 2$ bolometer, the cut waveguide reduces the thermal capacitance and the thermal conductance is the main loss. However, from the frequency response curve, the $3 \mathrm{~dB}$ bandwidth of the $\mathrm{B} 2$ bolometer is raised to higher than $1 \mathrm{kHz}$ (which is the frequency limit of the chopper in our setup). And the sensitivity is $0.87 \% / \mathrm{mW}$ at $20 \mathrm{~Hz}$. In the frequency response curve of the B1S bolometer the response first decays slightly with frequency before increasing again. The mechanism is unknown and more data in a higher frequency range is required. The sensitivity of $\mathrm{B} 1 \mathrm{~S}$ at $20 \mathrm{~Hz}$ is $1.56 \% / \mathrm{mW}$. The NEP decays with increasing chopper frequency. Once the frequency is above $200 \mathrm{~Hz}$, the noise tends to be constant with frequency up to $1 \mathrm{kHz}$.

In the B2 and B1S, the SNR is higher than $10 \mathrm{~dB} / \mathrm{mW}$. As above, the limitation of the noise measurement is the equipment, which is confirmed by the experimental measurements shown in supplement A. If either the noise in equiment is reduced or resistance of the bolometers is decreased, the SNR will be increased. Table 2 presents the performance parameters of the three bolometers at $400 \mathrm{~Hz}$. We confirmed that the change in current was due to a bolometric effect by measuring the responsivity when no bias current was applied. We found that a much weaker non-bolometric effect was present, which is discussed further in the supplementary information.

\section{Table 2: Performance Parameters of} Bolometer

\begin{tabular}{llll}
\hline Bolometer & B1 & B2 & B1S \\
\hline Resistance $(\mathrm{M} \Omega)$ & 60 & 152 & 656 \\
Sensitivity & $0.17 \pm$ & $0.80 \pm$ & $1.13 \pm$ \\
$(\% / \mathrm{mW})$ & 0.01 & 0.03 & 0.04 \\
SNR $(/ \mathrm{mW})$ & 232.69 & 696.96 & 747.18 \\
NEP $(\mu \mathrm{W} / \sqrt{H z})$ & 90 & 49 & 66 \\
\hline
\end{tabular}




\section{Future Improvements}

Although this bolometer shows very promising performance, there are still several significant improvements that could be made. Firstly, by looking at equations 1 and 2 we see that if we consider the DC case when the frequency $\omega=0$, then the parameters that affect the responsivity for a fixed optical input power are the TCR, the optical absorption efficiency $\varepsilon$, and the thermal conductance $G_{t h}$.

We achieve a TCR in these ion-implanted aSi thermometer arms that is around two times lower than the values typically reported in literature for deposited a-Si:H thin films, therefore altering the fabrication process could improve the TCR.

The optical absorption efficiency in these bolometers is below 0.8 (since the lowest antenna transmission is $20 \%$, and there will also be some scattering and reflection), so there is some modest room for improvement of this parameter, which could be attained by optimising the antenna fabrication to more accurately achieve the targeted dimensions. Alternatively the use of perfect absorber geometry could be explored.29

The third parameter $G_{t h}$ can be estimated from equations 1 and 2 . If we use the measured TCR of $0.90 \%$, the best measured responsivity of $1.56 \% / \mathrm{mW}$, and an estimate of $\varepsilon=0.5$, we find that $G_{t h}$ is approximately $3 \times 10^{-4} \mathrm{~W} / \mathrm{K}$. This is highly encouraging, since in the amorphous silicon microbolometers used in camera arrays $G_{t h}$ values of $2 \times 10^{-8}$ $\mathrm{W} / \mathrm{K}$ have been reported, ${ }^{322}$ indicating that responsivity improvements of up to 4 orders of magnitude might be possible in future, which could enable waveguide integrated bolometers to measure nanowatt optical power levels. This could be achieved by cutting the highly thermally conducting connection to the c-Si input waveguide with an air gap to create a bolometer island, which would be supported only by the a-Si thermometer arms. The challenge would be to do this in a way that does not introduce high optical loss and preserves the mechanical stability.

Besides the responsivity there are other pos- sible areas of improvement: (i) the resistance could be reduced by introducing doping to the a-Si thermometer arms, (ii) the antenna material could be changed from Au to a CMOS compatible material (e.g. Al), (iii) the fabrication process could be redesigned to use a-Si thin film deposition so that the ion implantation step is not required, (iv) the plasmonic absorber could be redesigned for even more wideband absorption characteristics, and v) the read out circuit could be improved to reduce the noise floor. We see this as a rich area for further research.

\section{Conclusions}

In this work, we have designed and fabricated the first room temperature silicon waveguidebased bolometer. These bolometers use amorphous silicon which is created by Ge ion implantation as the thermometer. In the experiment, the TCR of the a-Si is $0.90 \pm 0.26 \% / K$. Since silicon is transparent up to $8 \mu \mathrm{m}$, the Au optical antennas are designed and placed on the surface of the waveguides as plasmonic absorbers. The fabricated dimensions of the antennas are larger than intended. However, the response of the antennas is relatively stable with antenna length. Some variation between antennas is attributed to small variations in the shape of fabricated antennas, which could result in differences in the optical response. Moreover, the measured response bandwidth of the antennas is wide enough to cover the whole laser wavelength range, $3.72 \mu \mathrm{m}$ to $3.88 \mu \mathrm{m}$, and have a simulated $3 \mathrm{~dB}$ absorption bandwidth of 640 nm.

In these fabricated bolometers, there are designs with two different geometries. For the bolometer with a straight waveguide, B1, the sensitivity is $0.17 \pm 0.01 \% / \mathrm{mW}$ at $3.8 \mu \mathrm{m}$ with $400 \mathrm{~Hz}$ chopper frequency. In the second design, B2, the waveguide is terminated after the end of the bolometer, which reduces the thermal conductance and capacitance, resulting in a sensitivity improvement to $0.80 \pm 0.03$ $\% / \mathrm{mW}$ at $400 \mathrm{~Hz}$. The B1 bolometers were also suspended by underetching the waveguide to decrease the heat loss from thermal conduc- 
tance to the surroundings, which increased the sensitivity to $1.13 \pm 0.04 \% / \mathrm{mW}$. The $3 \mathrm{~dB}$ frequency bandwidth of the B1 bolometer was only $142 \mathrm{~Hz}$, while for the other two bolometers types, the $3 \mathrm{~dB}$-bandwidth is larger than $1 \mathrm{kHz}$, which is limited by the chopper frequency. In addition, the noise equivalent power is down to $49 \mu \mathrm{W} / \sqrt{\mathrm{Hz}}$. However, the dominant noise source comes from the circuit, indicating that the bolometers themselves might have lower noise. We have identified several approaches that could be used to improve the sensitivity of these bolometers in future.

Because of the wide transparency range of the suspended Si platform, and because gold absorbs light strongly at all optical wavelengths, this detector design can be adapted to work at any wavelength in the transparency range of silicon, simply by re-optimising the waveguide and antenna geometry. Furthermore, with modifications of the fabrication process, similar bolometers could in future be integrated with almost any mid-infrared waveguide platform.

Acknowledgement Royal Academy of Engineering (M. Nedeljkovic fellowship RF201617/16/33); Engineering and Physical Sciences Research Council (EP/L01162X/1, EP/L021129/1, EP/N00762X/1).

\section{Supporting Information Avail- able}

FTIR measurement of polarisation dependent antennas array; noise measurements of bolometers; non-bolometric effect in bolometers.

\section{References}

(1) Mashanovich, G. Z.; Milosevic, M. M.; Nedeljkovic, M.; Cox, D.; Passaro, V. M.; Chong, H. M.; Soref, R. Mid-infrared silicon photonics for sensing applications. MRS Online Proceedings Library Archive 2012, $143 \%$.

(2) Soref, R. Mid-infrared photonics in silicon and germanium. Nature photonics $\mathbf{2 0 1 0}$, 4, 495 .

(3) Milošević, M. M.; Nedeljkovic, M.; Ben Masaud, T. M.; Jaberansary, E.; Chong, H. M.; Emerson, N. G.; Reed, G. T.; Mashanovich, G. Z. Silicon waveguides and devices for the mid-infrared. Applied Physics Letters 2012, 101, 121105.

(4) Soler Penadés, J.; Alonso-Ramos, C.; Khokhar, A.; Nedeljkovic, M.; Boodhoo, L.; Ortega-Moñux, A.; Molina-Fernández, I.; Cheben, P.; Mashanovich, G. Suspended SOI waveguide with sub-wavelength grating cladding for mid-infrared. Optics letters 2014, 39, 5661-5664.

(5) Gallacher, K.; Millar, R.; Griškevičiūte, U.; Baldassarre, L.; Sorel, M.; Ortolani, M.; Paul, D. Low loss Ge-on-Si waveguides operating in the 8 $14 \mu \mathrm{m}$ atmospheric transmission window. Optics express 2018, 26, 25667-25675.

(6) Brun, M.; Labeye, P.; Grand, G.; Hartmann, J.-M.; Boulila, F.; Carras, M.; Nicoletti, S. Low loss SiGe graded index waveguides for mid-IR applications. $O p$ tics express 2014, 22, 508-518.

(7) Osman, A.; Nedeljkovic, M.; Penades, J. S.; Wu, Y.; Qu, Z.; Khokhar, A.; Debnath, K.; Mashanovich, G. Suspended low-loss germanium waveguides for the longwave infrared. Optics letters 2018, 43, 5997-6000.

(8) Spott, A.; Peters, J.; Davenport, M. L.; Stanton, E. J.; Merritt, C. D.; Bewley, W. W.; Vurgaftman, I.; Kim, C. S.; Meyer, J. R.; Kirch, J.; Mawst, L. J.; Botez, D.; Bowers, J. E. Quantum cascade laser on silicon. Optica 2016, 3, 545-551.

(9) Sinobad, M.; Monat, C.; LutherDavies, B.; Ma, P.; Madden, S.; Moss, D. J.; Mitchell, A.; Allioux, D.; 
Orobtchouk, R.; Boutami, S.; Hartmann, J.-M.; Fedeli, J.-M.; Grillet, C. Mid-infrared octave spanning supercontinuum generation to $8.5 \mu \mathrm{m}$ in silicon-germanium waveguides. Optica 2018, 5, 360-366.

(10) Thomson, D.; Shen, L.; Ackert, J.; Huante-Ceron, E.; Knights, A.; Nedeljkovic, M.; Peacock, A.; Mashanovich, G. Optical detection and modulation at $2 \mu \mathrm{m}-2.5 \mu \mathrm{m}$ in silicon. Optics express 2014, 22, 10825-10830.

(11) Muneeb, M.; Vasiliev, A.; Ruocco, A.; Malik, A.; Chen, H.; Nedeljkovic, M.; Penades, J. S.; Cerutti, L.; Rodriguez, J.-B.; Mashanovich, G.; M. Smit, K.; Tourni, E.; Roelkens, G. III-V-on-siliconintegrated micro-spectrometer for the $3 \mu \mathrm{m}$ wavelength range. Optics express 2016, 24, 9465-9472.

(12) Wang, X.; Cheng, Z.; Xu, K.; Tsang, H. K.; Xu, J.-B. High-responsivity graphene/silicon-heterostructure waveguide photodetectors. Nature Photonics 2013, \%, 888.

(13) Qu, Z.; Nedeljkovic, M.; Wu, Y.; SolerPenadés, J.; Khokhar, A.; Cao, W.; Osman, A.; Qi, Y.; Aspiotis, N.; Morgan, K.; Huang, C. a. Waveguide integrated graphene mid-infrared photodetector. Silicon Photonics XIII. 2018; p $105371 \mathrm{~N}$.

(14) Huang, L.; Dong, B.; Guo, X.; Chang, Y.; Chen, N.; Huang, X.; Liao, W.; Zhu, C.; Wang, H.; Lee, C.; Ang, K.-W. Waveguide-integrated black phosphorus photodetector for mid-infrared applications. ACS nano 2018, 13, 913-921.

(15) Gassenq, A.; Gencarelli, F.; Van Campenhout, J.; Shimura, Y.; Loo, R.; Narcy, G.; Vincent, B.; Roelkens, G. GeSn/Ge heterostructure short-wave infrared photodetectors on silicon. Optics express 2012, 20, 27297-27303.
(16) Rogalski, A. Infrared thermal detectors versus photon detectors: I. Pixel performance. Material Science and Material Properties for Infrared Optoelectronics. 1997; pp 14-26.

(17) Wang, B.; Lai, J.; Li, H.; Hu, H.; Chen, S. Nanostructured vanadium oxide thin film with high TCR at room temperature for microbolometer. Infrared Physics 85 Technology 2013, 57, 8-13.

(18) Tissot, J.-L.; Rothan, F.; Vedel, C.; Vilain, M.; Yon, J.-J. LETI/LIR's amorphous silicon uncooled microbolometer development. Infrared Detectors and Focal Plane Arrays V. 1998; pp 139-145.

(19) Vedel, C.; Martin, J.-L.; OuvrierBuffet, J.-L.; Tissot, J.-L.; Vilain, M.; Yon, J.-J. Amorphous-silicon-based uncooled microbolometer IRFPA. Infrared Technology and Applications XXV. 1999; pp 276-284.

(20) Moreno, M.; Torres, A.; Ambrosio, R.; Kosarev, A. Bolometers; IntechOpen, 2012.

(21) Mahjouri-Samani, M.; Zhou, Y.; He, X.; Xiong, W.; Hilger, P.; Lu, Y. Plasmonicenhanced carbon nanotube infrared bolometers. Nanotechnology 2012, 24, 035502 .

(22) Sassi, U.; Parret, R.; Nanot, S.; Bruna, M.; Borini, S.; De Fazio, D.; Zhao, Z.; Lidorikis, E.; Koppens, F.; Ferrari, A. C.; Colli, A. Graphenebased mid-infrared room-temperature pyroelectric bolometers with ultrahigh temperature coefficient of resistance. Nature communications 2017, 8, 14311.

(23) Niklaus, F.; Vieider, C.; Jakobsen, H. MEMS-based uncooled infrared bolometer arrays: a review. MEMS/MOEMS technologies and applications III. 2008; p 68360D.

(24) Takeya, H.; Frame, J.; Tanaka, T.; Urade, Y.; Fang, X.; Kubo, W. Bolometric 
photodetection using plasmon-assisted resistivity change in vanadium dioxide. Scientific reports 2018, 8, 12764.

(25) Soler Penadés, J.; Ortega-Moñux, A.; Nedeljkovic, M.; Wangüemert-Pérez, J.; Halir, R.; Khokhar, A.; Alonso-Ramos, C.; Qu, Z.; Molina-Fernández, I.; Cheben, P.; Mashanovich, G. Z. Suspended silicon mid-infrared waveguide devices with subwavelength grating metamaterial cladding. Optics express 2016, 24, 22908-22916.

(26) Milosevic, M. M.; Chen, X.; Cao, W.; Runge, A. F.; Franz, Y.; Littlejohns, C. G.; Mailis, S.; Peacock, A. C.; Thomson, D. J.; Reed, G. T. Ion implantation in silicon for trimming the operating wavelength of ring resonators. IEEE Journal of Selected Topics in Quantum Electronics 2018, 24, 1-7.

(27) Chen, X.; Milosevic, M. M.; Khokhar, A. Z.; Thomson, D. J.; Reed, G. T. Optical Waveguides by Germanium Ion Implantation on Siliconon-Insulator Platform. Conference on Lasers and Electro-Optics/Pacific Rim. 2018; pp Th3C-3.

(28) Bharadwaj, P.; Deutsch, B.; Novotny, L. Optical antennas. Advances in Optics and Photonics 2009, 1, 438-483.

(29) Bruck, R.; Muskens, O. L. Plasmonic nanoantennas as integrated coherent perfect absorbers on SOI waveguides for modulators and all-optical switches. Optics express 2013, 21, 27652-27661.

(30) Février, M.; Gogol, P.; Aassime, A.; Mégy, R.; Delacour, C.; Chelnokov, A.; Apuzzo, A.; Blaize, S.; Lourtioz, J.M.; Dagens, B. Giant coupling effect between metal nanoparticle chain and optical waveguide. Nano letters 2012, 12, 1032-1037.

(31) Chen, C.; Mohr, D. A.; Choi, H.-K.; Yoo, D.; Li, M.; Oh, S.-H. Waveguide-
Integrated Compact Plasmonic Resonators for On-Chip Mid-Infrared Laser Spectroscopy. Nano letters 2018, 18, 7601-7608.

(32) Yon, J.-J.; Mottin, E.; Tissot, J.-L. Latest amorphous silicon microbolometer developments at LETI-LIR. Infrared technology and Applications XXXIV. 2008; p 69401W. 